\title{
Poster Session II. Generation
}

\section{OPACITY OF MONODISPERSE AEROSOLS IN DOWN-SCALE TRANSMISSOMETER}

\author{
W.Y. LIN', L.K. HUANG ${ }^{2}$, L.Y. CHEN ${ }^{2}$, C.C. CHEN ${ }^{3}$ and F.T. JENG ${ }^{2}$ \\ ${ }^{1}$ Department of Environmental Engineering, KungShan Institute of Technology, Tainan, Taiwan. \\ ${ }^{2}$ Graduate Institute of Environmental Engineering, National Taiwan University, Taipei, Taiwan. \\ ${ }^{3}$ Institute of Occupational Medicine and Industrial Hygiene College of Public Health, National Taiwan \\ University, Taipei, Taiwan.
}

Keywords: TRANSMISSOMETER, MONODISPERSE, EXTINCTION, OPACITY.

\section{INTRODUCIION}

Current researches on visibility reduction have focused on discussing the relationships between visible light transmission and extinction of aerosols directly. The purpose for this study is to survey the optical characteristics of aerosols and the opacity source corresponding to different aerosols in visible spectrum with experimental approach using a self-developed opacity meter. The results would help to figure out the contribution on light extinction by all observed manipulated variables of aerosols, including number concentration, size distribution, etc.

\section{METHODS AND RESULTS}

Transmissometry is an approach widely employed for estimating ambient aerosol extinction with less than $10 \%$ error (Malm 1991). Therefore, the experimental system in this study was set up based on the design philosophy of transmissometry. The whole experiment setup consisted of three sub-systems: aerosol generation, opacity measurement and particle size distribution measurement systems.

The challenged aerosols, made from a glycerin-alcohol solution, were generated by a vibrating orifice monodisperse aerosol generator (VOMAG, TSI Model 3450). The generated aerosol size distribution was a function of solution concentration and vibrating frequency. The aerosols were carried into a test chamber, where a He-Ne laser beam passing through to measure opacity and an aerodynamic particle sizer (APS, TSI Model 3310A ) connecting to measure aerosols. The He-Ne laser (at $633 \mathrm{~nm}$ ) with stable energy output was equipped to project through the test chamber filled with challenging aerosols. Due to the extinction effects of aerosols, the power of laser beam would decrease and was then measured by an energy meter. The calculation of opacity caused by aerosols is based on the measurements with manipulated laser powers. The resulted opacity were compared with theoretical counterparts calculated based upon Beer's Law and measured particle size distribution.

Two cases of monodisperse aerosols, 12.4 and $21.2 \mu \mathrm{m}$, were conducted. The results showed that theoretical opacity values were higher than the ones derived from laser energy readings (Figures 1 and 2). It could be related to the unconsidered multi-scattering effect and cross-sectional coverage of challenging aerosols.

There was a possibility that a photon encountered more than one scattering caused by aerosol particles before it reached the energy meter. The number of scattering events could become higher while the aerosi number concentration increased. However, it is assumed only single scattering event occurs in the Beer's law adopted in this study.

As the number concentration of aerosols increased, it was also important to consider the effect of crosssectional coverage by aerosols along the path of laser beam. Though it is assumed that particles act as 
independent scatters in Beer's law.

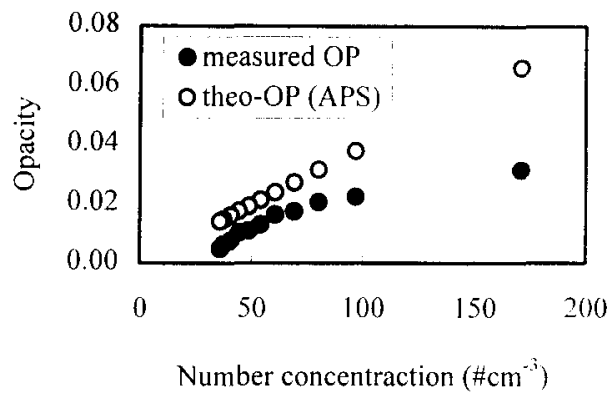

Figure 1. Opacity of $12.4 \mu \mathrm{m}$ aerosols.

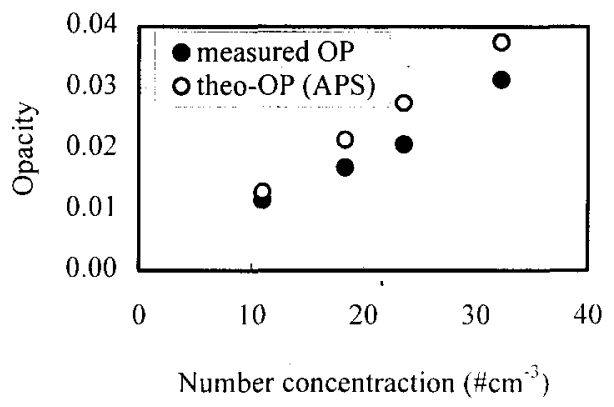

Figure 2. Opacity of $21.2 \mu \mathrm{m}$ aerosols.

\section{CONCLUSIONS}

An experimental system was established to investigate optical property of aerosols. Two cases of monodisperse glycerin aerosols were generated for size equal to 12.4 and $21.2 \mu \mathrm{m}$, respectively. The results showed measured opacity was lower than theoretical calculation. Multi-scattering and coverage effect of aerosols needed to be further studied to understand their effects to opacity.

\section{REFERENCES}

Reist, P. C. (1993), Aerosol Science and Technology, (McGraw-Hill, New York). Hasan, H. and Dzubay, T. G. (1983), Atmospheric Environment 17, 1573-1581.

Malm, W. C. (1991), Aerosol Science and technology 14, 459-471. 